\title{
Developing an Optimization Tool for Evaluating the Transmission System Expansion
}

\author{
T. F. Silva, A. N. Souza and P. da Costa Jr
}

\begin{abstract}
The transmission system is responsible for connecting the power generators to consumers safely and reliably, its constant expansion is necessary to transport increasing amounts of electricity. In order to help the power systems engineers, an optimization tool for optimize the expansion of the transmission system was developed using the modeling method of the linearized load flow and genetic. This tool was designed to simulate the impact of different scenarios on the cost of transmission expansion. The proposed tool was used to simulate the effects of the presence of distributed generation in the expansion of a fictitious transmission system, where it was found a clear downward trend in investment required for the expansion of the transmission system taking account of increasing levels of distributed generation.
\end{abstract}

Keywords - Power System Planning, Transmission Lines, Genetic Algorithms.

\section{INTRODUÇÃO}

$\mathrm{O}$ SISTEMA Elétrico Brasileiro é o maior da América Latina, composto por 134 usinas hidroelétricas, 93 usinas termoelétricas, 485 pequenas centrais hidroelétricas, 187 usinas a biomassa, 56 usinas eólicas e 1 usina solar, resultando em uma capacidade instalada de $115 \mathrm{GW}$ em 31/12/2011, se considerada a parcela de energia importada de Itaipu, conforme [1] e [2]. Em 2012, o consumo de eletricidade no Brasil cresceu 3,5\%, de acordo com dados da EPE divulgados em [3], influenciado sobretudo pelos crescimentos dos setores de comércio e serviços $(+7,9 \%)$ e residencial $(+5,0 \%)$, enquanto o setor industrial manteve-se estável.

Para acompanhar o crescimento da demanda, os sistemas de transmissão tem que ser constantemente ampliados, o que no Brasil é feito de forma integrada à expansão do parque gerador [2]. A Tabela Iapresenta a ampliação planejada do sistema de transmissão para o período entre 2011 e 2021, enquanto a Figura 1 apresenta os investimentos necessários para a construção das novas linhas.

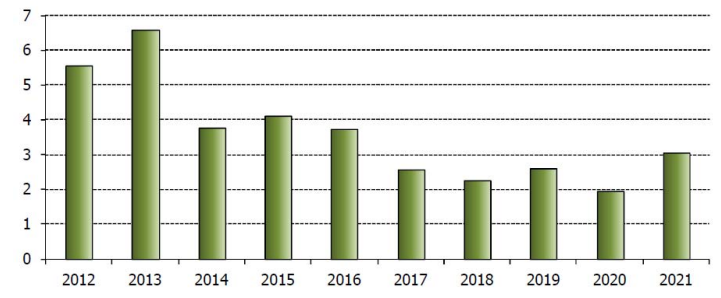

Figura 1. Investimentos previstos em linha de transmissão.

T. F. da Silva, Univ. Estadual Paulista (UNESP), Bauru, São Paulo, Brasil, tiago.forti@gmail.com

A. N. de Souza, Univ. Estadual Paulista (UNESP), Bauru, São Paulo, Brasil, andrejau@feb.unesp.br

P. da Costa Jr, Univ. Estadual Paulista (UNESP), Bauru, São Paulo, Brasil, costajr@feb.unesp.br
O planejamento do sistema de transmissão é realizado de acordo com programas computacionais especialistas, como o ANAREDE, por exemplo. Entretanto, em fases preliminares de um estudo, nem sempre todas as informações da rede estão disponíveis, como em testes de geração e demanda, onde os métodos de fluxo de carga não-lineares nem sempre conseguem convergir para uma solução, ou pode ser necessário analisar um grande número de cenários [4]. Nesses casos, uma ferramenta de otimização pode ser utilizada para estimar soluções para o problema da expansão da transmissão, com precisão aceitável, servindo como um método de pré-seleção, reduzindo o número de cenários que serão objetos de estudos mais profundos, como análises técnicas detalhadas, avaliações de impactos socioambientais, dentre outros.

TABela I. LinHAS DE TRANSMisSÃo PlaneJADAS ENTRE 2011 E 2021.

\begin{tabular}{ccccc}
\hline & $\begin{array}{c}\text { EXISTENT } \\
\mathrm{E}\end{array}$ & $\begin{array}{c}\text { PLANEJAD } \\
\mathrm{O}\end{array}$ & $\begin{array}{c}\text { PLANEJAD } \\
\mathrm{O}\end{array}$ & $\begin{array}{c}\text { PLANEJAD } \\
\mathrm{O}\end{array}$ \\
& 2011 & $2012-2016$ & $2017-2021$ & 2021 \\
\hline 800 & 0 & 0 & 7.325 & 7.325 \\
$\mathrm{kV}$ & & & & \\
750 & 2.683 & 0 & 0 & 2.683 \\
$\mathrm{kV}$ & & & & \\
600 & 1.612 & 4.750 & 0 & 6.362 \\
$\mathrm{kV}$ & & & & \\
500 & 34.851 & 21.547 & 5.342 & 61.740 \\
$\mathrm{kV}$ & & & & \\
440 & 6.679 & 47 & 66 & 6.792 \\
$\mathrm{kV}$ & & & & \\
345 & 10.063 & 337 & 0 & 10.400 \\
$\mathrm{kV}$ & & & & \\
230 & 45.349 & 7.874 & 444 & 53.668 \\
$\mathrm{kV}$ & & & & 148.969 \\
$\mathrm{Tota}$ & 101.237 & 34.555 & 13.177 & \\
1 & & & & \\
\hline
\end{tabular}

Um processo de otimização consiste em uma busca da melhor solução possível para um determinado problema, o ponto mínimo global do espaço de solução, respeitando as restrições do problema [5]. No caso da expansão do sistema de transmissão, isso significa encontrar a alternativa que apresente o menor investimento possível, respeitando as restrições do problema, como os limites de geração de cada usina e o fluxo nas linhas, por exemplo. Uma revisão bibliográfica compilando um grande número de técnicas utilizadas no planejamento da expansão da transmissão pode ser encontrada na referência [6]. A otimização é normalmente divida em duas partes, a modelagem do problema e a obtenção da solução ótima. Neste trabalho o método de modelagem escolhido foi o baseado no Fluxo de Carga Linearizado (ou Fluxo de Carga DC) e o processo de busca da solução escolhido foi o Algoritmo Genético. 


\section{A. Geração Distribuída}

As principais características do sistema elétrico tradicional, com a geração localizada em relativamente poucas unidades geradoras de grande porte e distantes dos grandes centros de consumo, demandando grandes quantidades de energia fluindo através de longas linhas de transmissão, com fluxo unidirecional da eletricidade, tem sido alvo de grandes críticas devido aos seus altos custos, perdas elevadas, grandes impactos ambientais e vulnerabilidades de segurança. Como alternativa a esses problemas, a geração distribuída (GD) de energia é considerada como uma tendência para os sistemas elétricos do futuro [6].

Como a implantação da geração distribuída ainda é pequena ou inexistente em muitos países, vários trabalhos estudam as políticas, desafios e incentivos governamentais para o seu desenvolvimento, como [7], [8] e [9], por exemplo. A influência da GD nos mercados de energia é analisada em [10] e [11], enquanto seu impacto nos sistemas de distribuição, em fatores como confiabilidade, controle, qualidade da energia e segurança do sistema, é verificado em [12], [13] e [14].

No Brasil, a geração distribuída ainda é uma perspectiva pro futuro, sendo que as leis e normatizações envolvendo o cenário de smart-grid ainda estão sendo desenvolvidas. Dessa maneira, é interessante analisar o impacto que a perspectiva da GD teria no planejamento da expansão dos sistemas de transmissão, especialmente aquela realizada por iniciativa dos consumidores, normalmente utilizando placas solares.

Para isso, foi escolhido um sistema de transmissão fictício, onde foram analisados 3 diferentes cenários. No cenário 1 , realiza-se a otimização do sistema de transmissão sem considerar a presença de geração distribuída, onde toda a energia consumida pelas cargas deve ser suprida pelas barras que possuem geradores conectados, servindo assim de base de comparação. No cenário 2, a otimização é feita considerando que a GD será responsável por suprir 1\% da carga do sistema, permitindo assim verificar qual será o impacto da GD nos investimentos necessários para a ampliação das linhas de transmissão. Por fim, no cenário 3, é considerado a presença de GD equivalente a $5 \%$ da carga, visando traçar uma tendência do impacto da GD nos custos do planejamento da transmissão.

\section{FERRAMENTA DESENVOLVIDA}

A ferramenta desenvolvida tem como objetivo a realização de testes envolvendo a otimização da expansão, facilitando a simulação de como os fatores de interesse impactam no custo do planejamento da transmissão. $\mathrm{O}$ objetivo durante seu desenvolvimento foi criar uma ferramenta robusta e de rápida implementação, sendo que o MATLAB foi utilizado na fase de implementação, por permitir um desenvolvimento rápido e simples, e apresentar uma grande flexibilidade de utilização.

O processo de desenvolvimento da ferramenta proposta está ilustrado em forma de fluxograma na Figura 5.1.

O primeiro passo para implementação da ferramenta de otimização da expansão da transmissão é a definição do circuito de teste, já que dependendo das características do circuito estudado, podem ocorrer modificações nas etapas seguintes, como nos objetivos da otimização e na função de avaliação.
Em seguida, devem ser estabelecidos os objetivos da otimização. O objetivo principal é sempre a minimização de uma função, que normalmente representa o custo da expansão do sistema. Entretanto, podem ser estabelecidos objetivos secundários para o problema, como por exemplo, avaliar o efeito de alocar mais ou menos geração para uma usina em particular, variar a carga de algumas barras, ou ainda analisar o impacto de fatores externos, como a influência da presença de geração distribuída, por exemplo.

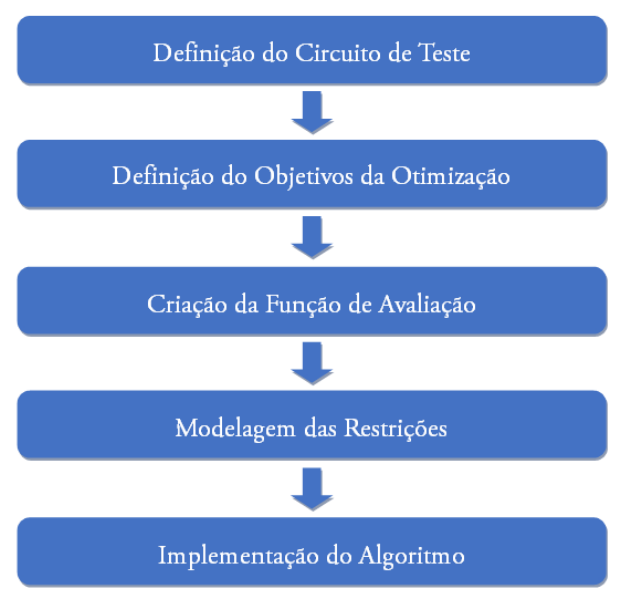

Figura 2. Desenvolvimento da ferramenta proposta.

Uma vez estabelecidos o circuito e os objetivos, a modelagem do problema é estabelecida com a criação da função objetivo e das restrições do problema. Neste trabalho, a modelagem foi realizada utilizando o método baseado no fluxo de carga linearizado.

Neste trabalho, cada linha de transmissão foi inserida com uma variável na função objetivo, onde seu valor era dado como o número de circuitos propostos pelo algoritmo, para ser em seguida multiplicado pelo custo de construção de cada circuito.

As restrições do problema são as equações que restringem o espaço de busca da solução, garantindo que as soluções encontradas sejam viáveis tecnicamente. Normalmente, as primeiras restrições referem-se a balanços de potência nas linhas e barras do sistema, limites de fluxo nas linhas e as capacidades de geração nas barras. Também podem ser incluídas nas restrições, equações referentes a objetivos secundários da otimização, como por exemplo, as condições de segurança do sistema, limites de tensão em determinadas barras, abertura angular da tensão entre pontos específicos do sistema, ou outros fatores que sejam de interesse.

Finalmente, a última etapa é a implementação do algoritmo, que deve procurar, dentro do espaço de busca, a solução que apresente o menor custo possível para a função objetivo, obedecendo as restrições do problema. Nesse momento, deve ser escolhido um método de busca para realizar a otimização da solução, o que neste trabalho foi realizado utilizando a técnica dos algoritmos genéticos.

\section{A. Fluxo de Carga Linearizado}

O fluxo de potência ativa em uma linha de transmissão é aproximadamente proporcional a abertura angular da tensão, se 
deslocando dos ângulos maiores para os ângulos menores. A relação entre os fluxos de potência ativa e as aberturas angulares é similar aquelas existente entre as tensões e correntes em um circuito de corrente contínua. Essa propriedade permite a formulação de um modelo aproximado, chamado de fluxo de carga CC ou fluxo de carga linearizado, que é utilizado para calcular a distribuição dos fluxos em um sistema de transmissão, com baixo custo computacional e uma precisão razoável [4].

Sabe-se que o fluxo de potência ativa $\left(P_{k m}\right)$ em uma dada linha de transmissão é dado por:

$$
\begin{aligned}
P_{k m}=V_{k}^{2} g_{k m}- & V_{k} V_{m} g_{k m} \cos \left(\theta_{k m}\right) \\
& -V_{k} V_{m} b_{k m} \operatorname{sen}\left(\theta_{k m}\right)
\end{aligned}
$$

Onde:

- $V_{k}=$ Módulo da tensão na barra $k$;

- $V_{m}=$ Módulo da tensão na barra $m$;

- $g_{k m}=$ Admitância da linha entre as barras $k$ e $m$;

- $\quad b_{k m}=$ Susceptância da linha entre as barras $k$ e $m$; e

- $\theta_{k m}=$ Abertura angular entre as barras $k$ e $m$;

Já o fluxo no sentido oposto $\left(P_{m k}\right)$ é calculado como:

$$
\begin{aligned}
P_{k m}=V_{k}^{2} g_{k m}-V_{k} & V_{m} g_{k m} \cos \left(\theta_{k m}\right) \\
& +V_{k} V_{m} b_{k m} \operatorname{sen}\left(\theta_{k m}\right)
\end{aligned}
$$

E as perdas na transmissão são:

$$
P_{k m}+P_{m k}=g_{k m}\left[V_{k}^{2}+V_{m}^{2}-2 V_{k} V_{m} b_{k m} \cos \left(\theta_{k m}\right)\right]
$$

Se os termos referentes as perdas forem desprezados, então teremos:

$$
P_{k m}=-P_{m k}=-V_{k} V_{m} b_{k m} \operatorname{sen}\left(\theta_{k m}\right)
$$

As seguintes aproximações podem ser feitas:

$$
\begin{aligned}
& V_{k} \cong V_{m} \cong 1 p u \\
& \operatorname{sen}\left(\theta_{k m}\right) \cong \theta_{k m} \\
& b_{k m} \cong \frac{-1}{x_{k m}}
\end{aligned}
$$

O fluxo de potência $P_{k m}$ pode então ser aproximado como:

$P_{k m}=x_{k m}^{-1} \theta_{k m}=\frac{\theta_{k}-\theta_{m}}{x_{k m}}$

Em que $x_{k m}$ é a reatância equivalente do conjunto de linhas em paralelo entre as barras $k$ e $m$.

O modelo linearizado também pode ser expresso na forma matricial, através de uma equação do tipo $I=Y \cdot E$. Partindo do fluxo de potência ativa:

$$
P_{k m}=x_{k m}^{-1} \theta_{k m}
$$

A injeção de potência ativa na barra $k$ é igual a soma do fluxos que saem da barra, ou seja:

$$
P_{k}=\sum x_{k m}^{-1} \theta_{k m}
$$

Essa equação pode ser escrita na forma:

$$
P_{k}=\left(\sum x_{k m}^{-1}\right) \theta_{k}+\sum\left(-x_{k m}^{-1} \theta_{m}\right)
$$

Que por sua vez admite uma representação matricial do tipo:

$$
P=B^{\prime} \cdot \theta
$$

Onde:

- $P=$ Vetor das injeções de potência ativa;

- $B^{\prime}=$ Matriz e admitância nodal; e

- $\theta=$ Vetor dos ângulos das tensões nodais;

Os termos da matriz $B^{\prime}$ são calculados como:

$$
B_{k m}^{\prime}=-x_{k m}^{-1}
$$

$$
B_{k k}^{\prime}=\sum x_{k m}^{-1}
$$

Como as perdas foram desprezadas, a matriz $B^{\prime}$ que aparece na equação (11) é singular, e a soma dos componentes de $P$ é nula, logo, a potência de uma barra qualquer pode ser obtida a partir da soma algébrica das demais. Para resolver esse problema, retira-se uma das equações do sistema, representado pela formulação matricial da equação (11) e adota-se a barra correspondente como barra slack $\left(\theta_{k}=0\right)$. Dessa forma, o sistema passa a ser não-singular com dimensão de NB-1 barras, e cujos ângulos podem ser determinados a partir das injeções de potência especificadas. Maiores detalhes sobre o fluxo de carga linearizado podem ser encontrada na referência [4].

\section{B. Modelagem do Problema}

A aplicação das equações do fluxo de carga linearizado ao problema da modelagem da expansão da transmissão, resulta no modelo denominado como Modelo Linearizado, ou Modelo CC. O modelo CC representa matematicamente a expansão da transmissão através das seguintes equações (14) a (20):

Minimize:

$$
v=\sum c_{k m} n_{k m}
$$

Sujeito à::

$$
S \times P+g=d
$$

Ou seja, o custo da expansão da transmissão, denominado de $v$, é o custo de construção de cada circuito entre as barras $k$ e $m$, dado por $c_{k m}$, multiplicado pelo número de circuitos propostos, representado por $n_{k m}$. O objetivo da otimização, é 
encontrar a solução que apresente o menor custo $v$ possível, o que representaria a alternativa de expansão da transmissão com o menor investimento necessário.

A restrição imposta pela equação (15) corresponde ao balanço de potência no sistema, onde:

- $S=$ Matriz de ligações entre barras;

- $f=$ Fluxos de potência nas linhas;

- $g=$ Gerações ativas; e

- $d=$ Demandas ativas.

$P_{k m}-x_{k m}^{-1}\left(n_{k m}^{0}+n_{k m}\right)\left(\theta_{k}-\theta_{m}\right)=0$

Onde:

- $\quad P_{k m}=$ Fluxo entre as barras $k$ e $m$;

- $x_{k m}=$ Reatância entre as barras $k$ e $m$;

- $n_{k m}=$ Quantidade final de linha entre as barras $k$ e $m$;

- $n_{k m}^{0}=$ Quantidade inicial de linhas entre as barras $k$ e $m$;

- $\theta_{k}=$ Ângulo de fase da tensão na barra $k$; e

- $\theta_{m}=$ Ângulo de fase da tensão na barra $m$.

Se analisarmos a equação (16), veremos que ela afirma que o fluxo de potência ativa $P_{k m}$ é equivalente ao inverso da reatância $x_{k m}$, multiplicado pela diferença entre os ângulos da tensão na barra $k\left(\theta_{k}\right)$, e na barra $m\left(\theta_{m}\right)$, o que é justamente a Equação 7 do fluxo de carga CC. A única diferença entre as duas equações, é a presença dos termos $n_{k m}$ e $n_{k m}^{0}$, que simplesmente representam que o fluxo $P_{k m}$ entre as duas barras, é a soma dos fluxos em todos os circuitos naquela linha de transmissão.

$$
\begin{aligned}
& \left|P_{k m}\right| \leq\left(n_{k m}^{0}+n_{k m}\right) \bar{P}_{k m} \\
& 0 \leq g \leq \bar{g} \\
& 0 \leq n_{k m} \leq \bar{n}_{k m} \\
& (k, m) \in \Omega, k \in \Gamma
\end{aligned}
$$

A equação (17) representa o limite de fluxo de potência ativa nas linhas, a equação (18) é o limite de geração das usinas, a equação (19) restringe o número de circuitos que podem ser adicionados a cada linha do sistema, e finalmente, a equação (20) define matematicamente o conjunto de trajetos possíveis para as linhas do sistema de transmissão, definido como $\Omega$, e o conjunto de barras do sistema, denominado de $\Gamma$.

Essas equações representam as informações básicas do problema, entretanto, outras restrições podem ser adicionadas a modelagem, dependendo do tipo de topologia do circuito, dos objetivos escolhidos para a otimização, dentre outros fatores. Mais informações sobre a modelagem baseada no fluxo de carga CC podem ser encontradas na referência [15].

\section{Algoritmos Genéticos}

Os algoritmos genéticos são baseados em uma analogia com a teoria da evolução, cujo conceito afirma que, considerando uma população de indivíduos qualquer, exposta a um ambiente com recursos limitados, os indivíduos melhores adaptados ao ambiente tem uma maior probabilidade de sobrevivência, consequentemente gerando mais descendentes, por outro lado, os indivíduos mal adaptados ao meio, tem uma chance menor de sobreviver, gerando menos descendentes. A combinação desses dois efeitos acaba por causar um aumento da aptidão média da espécie [16].

Levando essa analogia para o problema da expansão da transmissão, os indivíduos são as alternativas de ampliação do sistema de transmissão, onde a aptidão ao ambiente é medida pelo custo da alternativa, e pelo atendimento as restrições. Então a cada iteração do programa, é criada um novo conjunto de soluções possíveis, com o intuito de que o custo dessas soluções diminua.

O termo "algoritmos genéticos" representa uma classe de programas, que tem como objetivo a resolução de problemas complexos, combinando características de métodos de otimização determinísticos com elementos de técnicas heurísticas. Essa combinação torna os algoritmos genéticos (AGs) mais robustos do que os métodos de resolução direta, utilizando escolhas pseudo-aleatórias como uma ferramenta para guiar a procura da solução ótima por regiões do espaço de busca que seriam improváveis de outra maneira [16].

Conforme [17], as vantagens básicas dos algoritmos genéticos podem ser resumidas em:

- Trabalham com uma codificação do conjuntos de parâmetros, aumentando a robustez do método;

- Manipulam uma população de soluções possíveis, ao invés de um único ponto, diminuindo a probabilidade de mínimos locais; e

- Usam informação de custo da função objetivo, e não derivações ou outro tipo de dados auxiliares, diminuindo o tempo de processamento.

A principal desvantagem dos algoritmos genéticos consiste no fato de que não é possível provar matematicamente se o ponto encontrado é o ponto mínimo global. Entretanto, isso não configura um impedimento no caso da ferramenta proposta, já que o objetivo é utilizá-la em uma análise preliminar, onde as melhores alternativas seriam submetidas a fases subsequentes de análise, como estudos técnicos mais detalhados e análises de impacto socioambientais.

\section{Funcionamento da ferramenta}

O algoritmo implementado segue a estrutura lógica dos algoritmos genéticos, sendo que suas principais etapas estão descritas na Figura 3.

A primeira etapa do algoritmo é a criação da população inicial, que neste trabalho foi obtida de forma pseudo-aleatória. Em seguida inicia-se o processo iterativo, com a verificação das restrições do problema. Cada restrição que é violada gera um fator de penalidade para o indivíduo, que irá impactando negativamente no seu fitness. A terceira etapa do algoritmo é o cálculo do custo da função de avaliação, que é realizada para cada indivíduo da população atual. O valor do custo é combinado com as penalidades calculadas na etapa anterior, resultando nos valores de fitness dessa geração.

Com os valores de fitness devidamente calculados, são verificados os critérios de parada. Normalmente, se estipula um 
número máximo de gerações, para interromper a depuração do programa quando acontecem problemas de convergência. Como o algoritmo genético é uma técnica heurística, não é possível comprovar que o ponto mínimo global foi detectado. Para contornar essa dificuldade, estabelece-se como critério de parada um determinado número de gerações consecutivas sem melhora no fitness, o que indica que o algoritmo não está conseguindo encontrar pontos melhores no espaço de busca. Dependendo do problema estudado, ou do tipo de estratégia de algoritmo genético implementado, podem existir outros tipos de critérios de parada, entretanto, para a maioria das aplicações, esses dois critérios já são suficientes para garantir uma boa relação entre o tempo de processamento e a solução obtida.

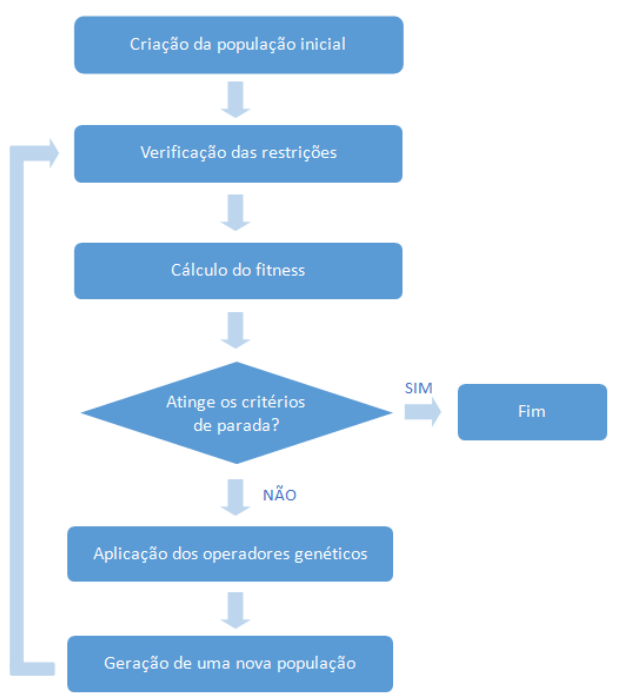

Figura 3. Funcionamento do algoritmo implementado.

Caso os critérios de parada tenham sido satisfeitos, o programa encerra o processamento e o melhor indivíduo da geração atual é eleito como a solução proposta para o problema. Do contrário, inicia-se a próxima etapa, que é a aplicação dos operadores genéticos, sendo as operações de crossover e mutação os mais comuns.

$\mathrm{Na}$ operação de crossover é atribuída um peso para cada indivíduo da população, de acordo com o seu fitness, representando a probabilidade de ser escolhido para gerar os descendentes da próxima geração. Na operação de mutação, alguns indivíduos são escolhidos pseudo-aleatoriamente para simular uma alteração na formação do código genético que não depende das características da geração anterior. Para tal, é selecionado um dos segmentos que formam o código do indivíduo, que tem o seu valor modificado pseudoaleatoriamente, para forçar a exploração de novas regiões do espaço de busca.

\section{EXPERIMENTO}

Para essa simulação, o circuito escolhido foi uma simplificação da região sudeste do SIN, apresentado na referência [18], possuindo 46 barras e 79 linhas de transmissão, como pode ser visto na Figura 4. Os valores de geração e consumo utilizados para cada barra do sistema estão compilados na Tabela II, representando uma demanda total de 6.800 MW. Todas as linhas de transmissão foram consideradas como existentes, com apenas 1 circuito cada, e o limite máximo estabelecido foi de 3 circuitos por linha de transmissão.

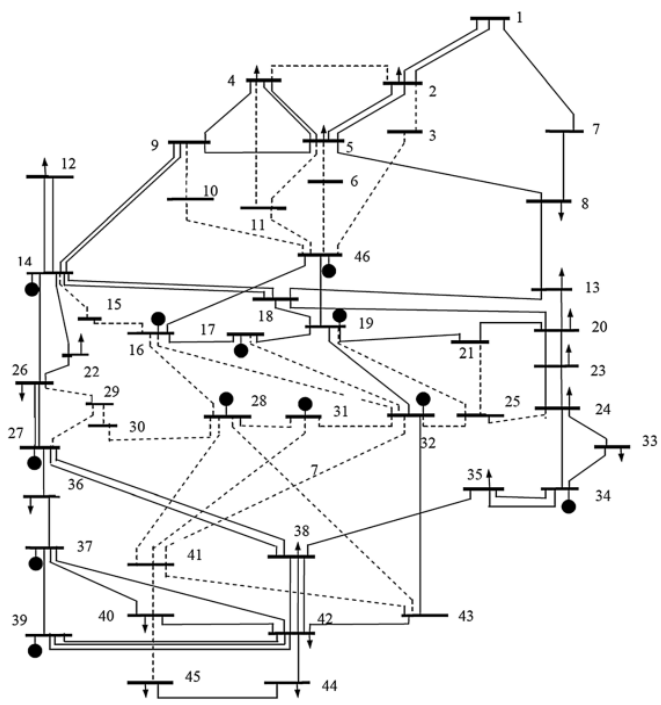

Figura 4. Circuito utilizado nos experimentos.

A proposta dos experimentos é realizar o processo de otimização da expansão do sistema de 46 barras, considerando nesse primeiro cenário que toda a energia demandada pelas cargas será suprida pelas barras de geração, como ocorre normalmente nos sistemas atuais, estabelecendo assim o investimento necessário na expansão do sistema. Em seguida, simula-se dois outros cenários, considerando que parte da carga será suprida através de geração distribuída, sendo essa quantidade equivalente a $1 \%$ da carga no cenário 2 , e $5 \%$ da carga no cenário 3 . Finalmente, comparando o custo encontrado nos cenários 2 e 3 , com a solução do cenário 1 , obtemos um indicativo do impacto que a geração distribuída tem sobre os investimentos em expansão do sistema de transmissão.

\section{A. Cenário 1}

No cenário 1 , é realizada uma rotina de otimização da expansão do sistema de transmissão de 46 barras, onde as barras de geração tem que suprir integralmente as cargas, sem nenhum nível de geração distribuída sendo considerado. Essa solução vai servir de base para as comparações com os valores obtidos nas simulações dos cenários 2 e 3 .

A solução encontrada pela ferramenta desenvolvida pode ser vista na Figura 5, onde cada variável do gráfico representa uma das linhas de transmissão do circuito. Pode-se verificar que a solução proposta é a construção de 2 circuitos adicionais nas linhas $3,4,11,46,47,73$ e 74, e 1 circuito adicional nas linhas 23 e 52 . Todas as demais linhas tem seus limites de fluxo dentro do permitido, sendo necessário apenas o circuito original.

$\mathrm{O}$ custo da melhor solução foi de 115.009 unidades monetárias, enquanto o custo médio da última geração, situouse em 123.589 unidades monetárias. 


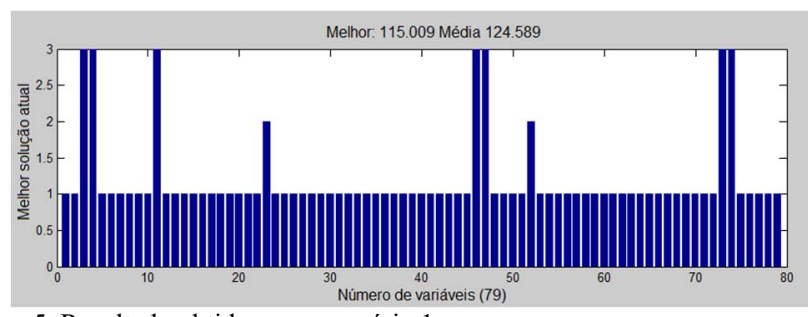

Figura 5. Resultado obtido para o cenário 1 .

\section{B. Cenário 2}

No cenário 2 , é considerado que $1 \%$ da carga será suprida por meio de geração distribuída. Foi considerado que em um cenário real, onde a GD seja economicamente atrativa para os consumidores, a sua implantação iria ocorrer obedecendo uma distribuição geográfica aleatória, portanto, para fornecer uma simulação mais realista de acordo com esse pondo de vista, a GD foi distribuída pelas barras proporcionalmente a carga existente na mesma, ao invés de ser alocada em apenas alguns poucos pontos do sistema.

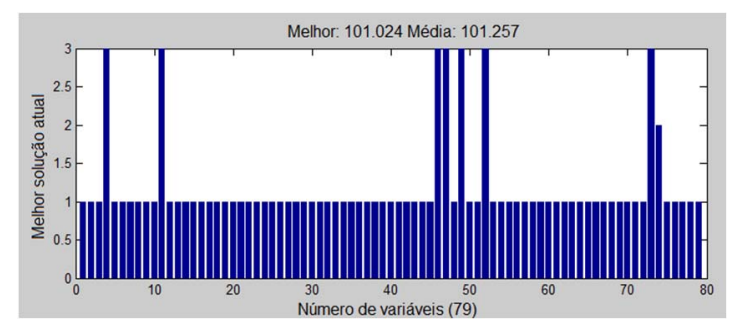

Figura 6. Resultado obtido para o cenário 2.

A solução obtida pode ser vista na Figura 6, onde cada variável do gráfico mostra o número de circuitos propostos em uma das linhas do sistema. O custo total da melhor solução foi de 101.024 unidades monetárias, o que representa uma redução de 13.985 u.m. (12,16\%) em comparação com o cenário 1. Isso significa que a presença de geração distribuída equivalente a $1 \%$ da carga, corresponderia para esse sistema uma economia de $12,16 \%$ no investimento necessário para a construção de novas linhas de transmissão. É importante salientar que essa porcentagem depende de vários fatores, como a topologia do circuito, quais usinas sofrerão redespacho, a presença ou não de congestionamento nas linhas, dentre outros.

\section{Cenário 3}

$\mathrm{O}$ terceiro cenário considera uma geração distribuída equivalente a $5 \%$ da carga total, sendo que foram adotadas as mesmas premissas do cenário 2, sobre a distribuição da GD ao longo do sistema, para manter a coerência dos experimentos. A solução obtida é apresentada na Figura 7.

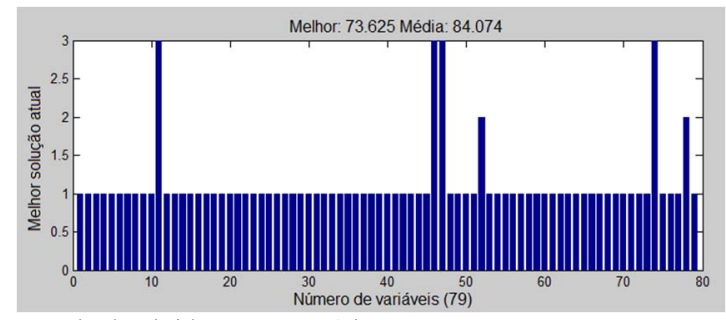

Figura 7. Resultado obtido para o cenário 3.
Neste cenário, o custo da melhor solução foi de 73.625 unidades monetárias, o que corresponde a uma redução de 41.384 u.m. (35,98\%) quando comparado ao caso base, enquanto o custo médio da última geração foi de 84.074 u.m.

\section{Resultados Obtidos}

Foram realizadas três rotinas de otimização utilizando o mesmo sistema de transmissão fictício, onde a única diferença foi a simulação de geração distribuída nos centros de carga.

Com os investimentos necessários em cada um dos cenários, para a expansão do sistema de transmissão fictício utilizado nos testes, é possível estimar uma tendência do impacto da geração distribuída nos custos do planejamento da expansão da transmissão. Essa comparação entre os custos é realizada na Tabela .

TABELA II. RESUMO DOS RESUltados OBTIDOS.

\begin{tabular}{ccccc}
\hline Cenário & $\begin{array}{c}\text { Geração } \\
\text { Distribuída }\end{array}$ & $\begin{array}{c}\text { Custo da } \\
\text { Expansão }\end{array}$ & $\begin{array}{c}\text { Economia } \\
\text { (u.m.) }\end{array}$ & $\begin{array}{c}\text { Economia } \\
(\%)\end{array}$ \\
\hline 1 & 0 & 115.009 & - & - \\
2 & $1 \%$ & 101.024 & 13.985 & $12,16 \%$ \\
3 & $5 \%$ & 73.625 & 41.384 & $35.98 \%$ \\
\hline
\end{tabular}

Percebe-se que existe uma clara tendência de redução do custo de expansão do sistema de transmissão em resposta a níveis crescentes de geração distribuída, o que é condizente com o fato de que a GD representa uma quantidade significativa de energia gerada próxima à carga, reduzindo os fluxos necessários no sistema de transmissão.

É importante salientar que essa porcentagem depende de vários fatores, como a topologia dos circuitos de transmissão, a ocorrência de sobrecarregamento na rede, a distribuição geográfica da geração distribuída, dentre muitos outros. Se considerarmos o tipo mais básico de geração distribuída, como a geração fotovoltaica, os consumidores arcam com os custos do equipamento e da sua instalação. $O$ investimento governamental é relativamente pequeno, consistindo basicamente de incentivos fiscais, isenções tributárias e linhas de crédito especiais. Como demonstrado aqui, uma parte considerável para os investimentos governamentais pode vir justamente da economia que será obtida na expansão do sistema de transmissão. Além disso, a redução do número de linhas de transmissão construídas apresentam outros benefícios, como menos perdas no transporte da energia elétrica e menores impactos socioambientais. Uma análise sobre o impacto ambiental dos sistemas de transmissão podem ser encontrada na referência [19].

\section{CONCLUSÃO}

Neste trabalho foi desenvolvida uma ferramenta de otimização da expansão da transmissão para as fases preliminares de planejamento, etapas em quê nem sempre todas as informações da rede estão disponíveis, podendo resultar em não-convergência dos métodos de fluxo de carga não-lineares, como em situações de testes de geração e demanda, por exemplo, além de normalmente ser necessário analisar um grande número de cenários. A ferramenta desenvolvida tem 
ainda como objetivo ser simples e abrangente, podendo colaborar no estudo do impacto de diversos fatores no custo da expansão do sistema de transmissão.

Após seu desenvolvimento e validação, a ferramenta proposta foi utilizada para simular o impacto da presença de geração distribuída no custo da expansão de um sistema de transmissão fictício. Para isso foram realizados testes com três diferentes cenários. O cenário 1 não contemplava a presença de geração distribuída, o segundo simulava a presença de geração distribuída equivalente a $1 \%$ da carga consumida, e o terceiro cenário estabelecia que $5 \%$ do consumo seria suprido pela geração distribuída.

Os resultados obtidos indicaram uma clara tendência de economia nos investimentos necessários para a expansão do sistema de transmissão, em resposta ao aumento da oferta de geração distribuída, resultando em economias da ordem de $12 \%$ e $36 \%$ para os cenários com $1 \%$ e $5 \%$ de geração distribuída, respectivamente. Esses resultados são coerentes com o fato de que uma porcentagem significativa da energia consumida seria produzida próxima a carga, através da geração distribuída, não reduzindo o fluxo necessário através do sistema de transmissão.

Podemos concluir que a ferramenta proposta apresentou resultados promissores, não tendo problemas de convergência ou de confiabilidade dentro do escopo dos testes realizados. Pretende-se aumentar a complexidade dos testes e circuitos utilizados nos trabalhos futuros. Desenvolver a ferramenta de forma a permitir a utilização de outras técnicas de busca e modelagens também é uma perspectiva promissora de trabalho.

\section{REFERÊNCIAS}

[1] Empresa de Pesquisa Energética - EPE, Plano Decenal de Expansão da Energia 2021, 2012.

[2] F. P. M. P. a. B. B. L. A. Barroso, "Solving the major challenges in transmission asset investment in the competitive environment: The brazilian case," Power Energy Society General Meeting, 2009.

[3] Empresa de Pesquisa Energética - EPE, "Consumo de energia elétrica no comércio cresceu 7,9\% em 2012," Resenha Mensal do Mercado de Energia Elétrica, Janeiro 2012.

[4] A. Monticelli, Fluxo de Carga em Redes de Energia Elétrica, 1983.

[5] L. V. S. Boyd, Convex Optimization, 2004.

[6] J. Molina e H. Rudnick, "Transmission of Electric Energy: a Bibliographic Review," IEEE Latin America Transactions, vol. 8, pp. pp.245,258, 2010.

[7] J. F. Z.-Y. D. a. K.-P. W. Jun Hua Zhao, "Flexible transmission network planning considering distributed generation impacts," 2011.

[8] D. B. C. H. D. J. a. M. S. Peter Dondi, "Network integration of distributed power generation," vol. Proceedings of the Seventh Grove Fuel Cell Symposium, 2002.

[9] S. M. a. B. Singh, "Distributed generation: Basic policy, perspective planning, and achievement so far in India.," 2009.

[10] J. Paska, "Distributed generation and renewable energy sources in poland," 2009.

[11] F. Gulli, "Small distributed generation versus centralised supply: a social cost-benefit analysis in the residential and service sectors," 2006.

[12] Y. X. a. G. B. J.A. Momoh, "An approach to determine distributed generation benefits in power networks," 2008.
[13] M. d. S. a. A. R. A.C. Neto, "Impact of distributed generation on reliability evaluation of radial distribution systems under network constraints," 2006.

[14] V. S. J. N. M. L. a. B. S. M. P. R. Khatri, "Improving power quality by distributed generation," 2005.

[15] R. M. a. P. Sivakumar, "Improving the power quality performance for distributed power generation," 2012.

[16] A. M. A. G. a. S. H. Romero R., "Test system and mathematical models for transmission network expansion planning," IEEE Proceedings of Generation, Transmission and Distribuition, 2002.

[17] Z. Michalewicz, Genetic Algorithms + Data Structures = Evolution Programs, Springer, 1999.

[18] D. E. Goldberg, Genetic Algorithms in Search, Optimization, and Machine Learning, New York: Addison Wesley Publishing Company, 1989.

[19] A. M. A. G. J. M. a. R. R. S. Haffner, "Branch and bound algorithm for transmission system expansion planning using a transportation model," 2000.

[20] R. S. J. a. E. G. Hertwich, "Environmental evaluation of power transmission in Norway," 2013.

[21] Empresa de Pesquisa Energética - EPE, Anuário Estatístico de Energia Elétrica, 2012.

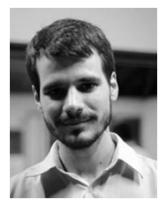

Tiago Forti da Silva é graduado em Engenharia Elétrica pela Universidade Paulista e possui título de mestre em Engenharia Elétrica pela Universidade Estadual Paulista Júlio de Mesquita Filho. Atualmente é professor da Universidade Paulista, e tem interesse pelos seguintes temas: sistemas elétricos de potência, otimização e modelagem matemática.

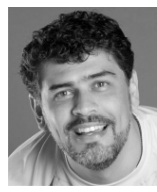

André Nunes de Souza é graduado pela Universidade Mackenzie em engenharia elétrica e possui títulos de Mestre e Doutor pela Escola Politécnica da Universidade de São Paulo (EPUSP). Atualmente é Professor Livre Docente da Universidade Estadual Paulista (UNESP), da Faculdade de Engenharia de Bauru, e coordena o Laboratório de Sistemas de Potência e Técnicas

Inteligentes (LSISPOTI), onde faz pesquisas em temas como sistemas inteligentes, eficiência energética, perdas comerciais, detecção de faltas em cabos subterrâneos, diagnóstico de pára-raios e qualidade de energia.

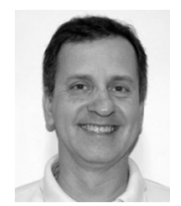

Pedro da Costa Junior é graduado em engenharia elétrica pela UNESP - Universidade Estadual Paulista, mestre e doutor em engenharia elétrica pela UNICAMP - Universidade Estadual de Campinas. Atualmente é professor assistente doutor II na UNESP - Universidade Estadual Paulista. Possui experiência na área de Engenharia Elétrica, com ênfase em Geração, Transmissão e Distribuição de Energia Elétrica, atuando principalmente com sistemas inteligentes, transitórios eletromagnéticos, modelagem de sistemas elétricos, perdas comerciais, detecção de faltas em sistemas de distribuição de energia, e sistemas de aterramento. 